\title{
El mapa de competencias a adquirir
}

Arcadi Gual

\section{Modelos docentes}

En los últimos diez años, la aparición de la palabra 'competencia/s' en el mundo universitario se ha convertido en un talismán capaz de arreglarlo todo. Los currículos, los planes de estudios, la programación docente, la evaluación por competencias, incluso Bolonia, se asocian al paradigma de la competencia. Pero, realmente, ¿̇son las competencias la solución a la docencia universitaria?, ¿son tan importantes como para que nos pasemos el día hablando de ellas? Las respuestas sencillas, un sí o un no, son útiles para simplificar, pero en este caso no son la mejor respuesta. Por ello, para responder si las competencias son especialmente relevantes en la formación universitaria, debemos empezar la reflexión desde otro lugar. Si recordamos cómo enseñamos cuando bajamos al aula y lo hacemos desde un punto de vista simple y esquemático nos encontraremos básicamente con dos modelos opuestos, el expositivo y el funcional, y por supuesto, con todos los niveles intermedios imaginables que son posibles.

\section{Modelo expositivo}

El modelo expositivo es el de siempre, el de toda la vida, el 'yo hablo y tú escuchas'. Yo sé lo que hay que saber y tú tomas nota de mi sabiduría y aprendes lo que puedas de mí, de mi libro, de mis apuntes o de mi powerpoint, que es la última de las perversiones docentes, no en sí misma sino en su uso. Y si lo que te pregunto en el examen no tiene nada que ver con lo que necesitas saber, pues tampoco importa mucho; la vida ya te enseñará. Este modelo, el expositivo, en lugar de definirlo con expresiones provocadoras como las anteriores, se puede definir con palabras más amables. Así, la tabla I resume las características fundamentales de este modelo, entre las que destaca, por encima de las demás, la característica de basarse en establecer de la forma más concreta posible los contenidos. Precisamente, los contenidos de la asignatura son los que permiten hacer el programa de la asignatura o incluso un libro de texto de la materia. En este modelo, yo, profesor que poseo el conocimiento, tengo la autoridad para determinar qué incluyo y qué no incluyo y, por supuesto, qué debe saber el alumno. Un innumerable grupo de profesores universitarios han construido sus memorias docentes en base a objetivos educativos o educacionales descritos y recomendados por la Organización Mundial de la Salud (OMS), según el trabajo realizado por Gilbert en los años cincuenta, y que no son más que una redacción del programa en objetivos educativos; esta clasificación se basaba en tres grandes grupos de objetivos educativos, los generales, los intermedios y los específicos o 'instruccionales'. Aunque son de utilidad al alumno, todos ellos van dirigidos al profesor, dado que en el modelo expositivo el centro lo ocupa el maestro. La conocida 'taxonomía de Bloom', fruto del trabajo coordinado por Benjamin Bloom y publicado en el año 1956, también fue una obra de referencia para pormenorizar un programa docente; concretamente definía tres grandes ámbitos: el cognoscitivo, que fue el mejor desarrollado y que abarcaba diferentes áreas: el psicomotor, que definía diferentes destrezas, y el afectivo, referido a las actitudes, valores y conductas. La taxonomía de Bloom se ha actua-
Catedrático de Fisiología. Facultad de Medicina. Universitat de Barcelona. Director de la Fundación Educación Médica.

E-mail

agual@ub.edu

Nota

Este manuscrito ha sido posible gracias a la idea y maestría de la Dra. Wilma Penzo, de la Facultad de Medicina de la Universitat de Barcelona, por lo que le estoy inmensamente agradecido. Igualmente agradezco a mis compañeros de la Fundación Educación Médica, el Dr. Albert Oriol Bosch, la Dra. María Nolla y el Dr. Jorge Palés, tanto la lectura crítica del manuscrito como sus aportaciones a éste. 


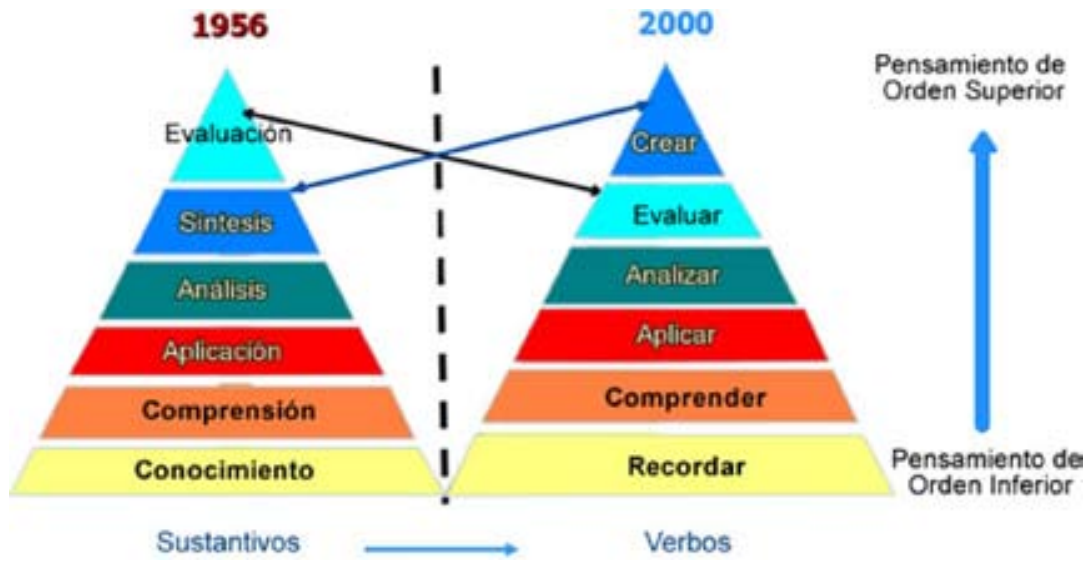

Figura 1. Cambios en la pirámide de la taxonomía de Bloom.

Tabla I. Características de los modelos expositivo y funcional.

\begin{tabular}{ll}
\hline Modelo expositivo & Modelo funcional \\
\hline Se basa en los contenidos & Se basa en el uso de los contenidos \\
Su meta es adquirir conocimientos & Su meta es aplicar los conocimientos \\
Concepto dogmático del 'saber' & El 'saber' depende del contexto \\
$\begin{array}{l}\text { Metodologías docentes y evaluativas } \\
\text { sin estrecha relación }\end{array}$ & $\begin{array}{l}\text { Metodologías docentes y evaluativas } \\
\text { con estrecha relación }\end{array}$ \\
Evaluación fácil y cómoda para el docente & Evaluación sobre actividades reales \\
\end{tabular}

lizado en dos revisiones posteriores: la primera, de Lovin y Anderson en el año 2001 bajo el título de 'Revisión de la taxonomía de Bloom', y la segunda, de Andrew Churches en el año 2008 bajo el título de 'Taxonomía digital de Bloom'. Las revisiones citadas incorporan cambios nada menores que le han añadido actualidad y valor conceptual, entre los que destaca el cambio de sustantivos por verbos (Fig. 1). Estas revisiones incorporan para cada nivel conceptual los verbos (Tabla II) y, por tanto, las acciones que les son propias. Esta modificación de clasificación debe interpretarse como un paso hacia el modelo funcional que fija cada vez más su mirada en el alumno antes que en el profesor.

\section{Modelo funcional}

El modelo funcional se acerca mucho más a la práctica, al mundo real, y busca que el aprendiz aprenda a hacer lo que tiene que hacer dejando en un segundo plano el conocimiento por el conocimiento. El modelo funcional no pretende en ningún momento quitar relevancia al conocimiento; lo que pretende, entre otras características (Tabla III), es priorizar el uso de los contenidos por encima de los propios contenidos. De esta manera, el contexto es protagonista al convertirse en pieza relevante del proceso. El estudiante aprende lo que necesita aplicar aquí y ahora. El modelo funcional se orienta en la misma dirección que la educación 
Tabla II. Revisión de la taxonomía de Bloom (modificado de Anderson y Krathwohl, 2000).

\begin{tabular}{|c|c|c|c|c|c|c|}
\hline & Recordar & Comprender & Aplicar & Analizar & Evaluar & Crear \\
\hline Descripción & $\begin{array}{l}\text { Reconocer } \\
\text { y traer a la } \\
\text { memoria } \\
\text { información } \\
\text { relevante de } \\
\text { la memoria } \\
\text { de largo plazo }\end{array}$ & $\begin{array}{l}\text { Habilidad } \\
\text { de construir } \\
\text { significado } \\
\text { a partir de } \\
\text { material } \\
\text { educativo, } \\
\text { como la } \\
\text { lectura o las } \\
\text { explicaciones } \\
\text { del docente }\end{array}$ & $\begin{array}{l}\text { Aplicación de } \\
\text { un proceso } \\
\text { aprendido, } \\
\text { ya sea en } \\
\text { una situación } \\
\text { conocida o } \\
\text { en una nueva }\end{array}$ & $\begin{array}{l}\text { Descomponer } \\
\text { el conocimiento } \\
\text { en sus partes y } \\
\text { pensar en cómo } \\
\text { éstas se relacionan } \\
\text { con su estructura } \\
\text { global }\end{array}$ & $\begin{array}{l}\text { Consta de } \\
\text { comprobación } \\
\text { y crítica }\end{array}$ & $\begin{array}{l}\text { Involucra reunir } \\
\text { cosas y hacer } \\
\text { algo nuevo. } \\
\text { Para llevar a } \\
\text { cabo tareas } \\
\text { creadoras, los } \\
\text { aprendices } \\
\text { generan, } \\
\text { planifican } \\
\text { y producen }\end{array}$ \\
\hline $\begin{array}{l}\text { Verbos } \\
\text { indicadores } \\
\text { de procesos } \\
\text { cognitivos }\end{array}$ & $\begin{array}{l}\text { Reconocer } \\
\text { Recordar } \\
\text { Listar } \\
\text { Describir } \\
\text { Recuperar } \\
\text { Denominar } \\
\text { Localizar }\end{array}$ & $\begin{array}{l}\text { Interpretar } \\
\text { Ejemplificar } \\
\text { Clasificar } \\
\text { Resumir } \\
\text { Inferir }\end{array}$ & $\begin{array}{l}\text { Ejecutar } \\
\text { Implementar } \\
\text { Desempeñar } \\
\text { Usar }\end{array}$ & $\begin{array}{l}\text { Diferenciar } \\
\text { Organizar } \\
\text { Atribuir }\end{array}$ & $\begin{array}{l}\text { Comprobar } \\
\text { Criticar } \\
\text { Revisar } \\
\text { Formular } \\
\text { hipótesis } \\
\text { Experimentar } \\
\text { Juzgar } \\
\text { Probar } \\
\text { Detectar } \\
\text { Monitorizar }\end{array}$ & $\begin{array}{l}\text { Planear } \\
\text { Generar }\end{array}$ \\
\hline
\end{tabular}

basada en resultados (outcome-based education). El planteamiento es, pues, bien distinto al de la educación del modelo expositivo, basada en el programa de la materia o asignatura. Allí enseñaré a los alumnos los contenidos que alguien, un experto, ha incluido en el programa; aquí construiré el programa en base a los resultados que la sociedad demanda que tenga el alumno al final del aprendizaje. Aquí estoy obligado a definir primero lo que quiero que el alumno aprenda y con esos resultados podré empezar a construir el programa. Aquí el centro es el alumno.

\section{Educación basada en resultados}

La educación basada en resultados no representa un modelo nuevo ya que fue propuesto hace más de dos décadas por William G. Spady como la necesidad de definir lo que se espera que los estudiantes dominen, comprendan y demuestren que saben hacer después de un periodo de aprendizaje (Spady, 1988). En este planteamiento, el producto que queremos define y determina el currículo y su organización, los métodos de enseñanza y las estrategias educativas, las asignaturas o materias a incluir, los procesos de evaluación, el entorno educativo y la distribución curricular. Naturalmente no sólo queda incluida la evaluación del alumno, sino también la evaluación del currículo. La consecuencia final es que la educación basada en resultados finales permite establecer el mejor diseño curricular, entendido éste no como el programa o las asignaturas, sino todo el conjunto de elementos de un diseño curricular entre los que deben considerarse, como mínimo, ocho elementos: las necesidades formativas, los resultados de aprendizaje, la estrategia educativa, los contenidos, las metodologías docentes, la evaluación, el entorno educativo y los procesos de gestión y revisión del currículo (Palés, 2006).

\section{Elementos de la planificación}

En el modelo expositivo, el alumno debe examinarse del programa fijado de antemano. Para facilitar su tarea, el programa de la asignatura, que 
Tabla III. Características y consecuencias de la educación basada en resultados.

\author{
Características
}

Pone énfasis en el producto final

Ayuda a distinguir lo que es esencial y relevante

Determina lo que se ha de enseñar y evaluar

Permite definir la responsabilidad del alumno, del profesor y de la institución respecto a la sociedad

Consecuencias

Organización y contenido del programa formativo

Estrategias y metodologías docentes

Recursos

Evaluación de los discentes y del programa

frecuentemente se parece al índice de un libro de texto, se acompaña de un listado de objetivos de aprendizaje, útiles especialmente para el profesor. Estos objetivos son una concreción del programa en puntos específicos y no deben identificarse con los objetivos finales propios de lo que denominamos 'educación basada en resultados'.

A diferencia del modelo expositivo, la enseñanza basada en resultados de aprendizaje está sujeta a un circuito de retroalimentación (Fig. 2). Las necesidades de formación comprometen los resultados de aprendizaje; éstos, a las metodologías, estrategias y actividades de aprendizaje, que a su vez condicionarán la evaluación. Y los resultados de la evaluación condicionarán de nuevo las necesidades de formación.

Es pues necesario diferenciar estos dos modelos de enseñanza y sus elementos consustanciales: el modelo expositivo se centra en el profesor y requiere un programa, mientras que el funcional se centra en el alumno y requiere unos objetivos finales que condicionan la estrategia, la metodología y la evaluación.

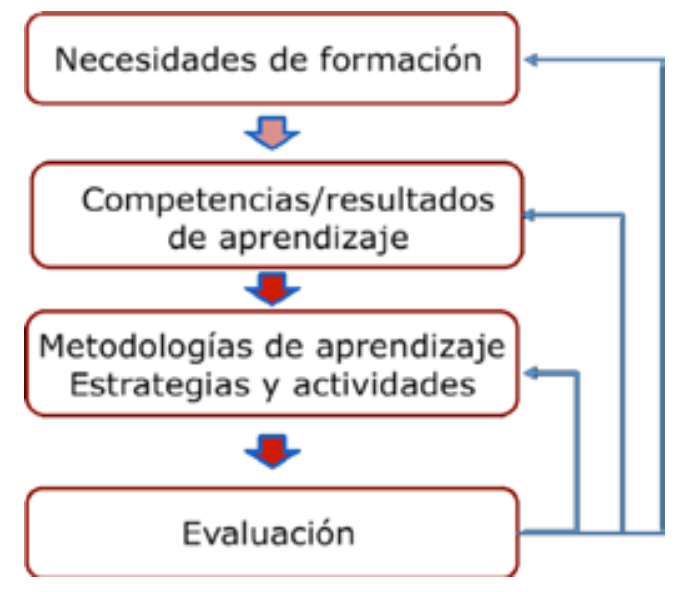

Figura 2. Elementos clave de la planificación.

\section{Competencias}

Las competencias que debe tener un estudiante al final de un proceso de aprendizaje -llámese grado, máster, posgrado o formación especializadase denominan por lógica 'competencias finales' y son las que el estudiante que ha cursado con éxito los estudios debe poder demostrar al terminarlos. Posiblemente, las competencias finales -o simplemente competencias- no son de forma exacta los resultados de aprendizaje que hemos definido en la enseñanza basada en resultados finales; quizá éstos sean un poco más concretos y de más fácil redacción, pero en cualquier caso se le parecen mucho. Es más, no creo que sea bueno querer diferenciarlos. Por ello, si bien ya he dicho que no son sinónimos, podemos y debemos utilizar como equivalentes los términos 'educación funcional', 'educación basada en objetivos finales', 'outcome-based education' o 'educación basada en competencias'.

¿Y dónde está el problema? Desgraciadamente, problemas hay varios, pero algunos son especialmente importantes; de éstos señalaré dos. El primero es que nos pasamos el día hablando de competencias mientras que el modelo más difundido y el mayoritariamente utilizado en nuestras aulas durante el curso académico 2010-2011 es el modelo expositivo, basado en el programa y, en todo caso, en la descripción de los objetivos de aprendizaje, y para el cual no se necesita ni definir competencias ni resultados de aprendizaje. 


\section{Tabla IV. Ejemplos de competencias descritas en el plan del grado de Medicina: tan sólo la primera puede considerarse una competencia.}

Diagnosticar y orientar el manejo de

las principales patologías cardiovasculares

Hacer una punción venosa

Embarazo y parto normal y patológico

Plantear y proponer medidas preventivas

adecuadas a una situación clínica

Vacunas

Epidemiología

Cicatrización

Prescripción y farmacovigilancia

Diagnóstico y consejo genético

a Orden ECI/332/2008, de 13 de febrero, por la que se establecen los requisitos para la verificación de los títulos universitarios oficiales que habiliten para el ejercicio de la profesión de médico.

El segundo problema es que no utilizamos el término 'competencia' de una forma unívoca. Quizá el tema es peor porque existen argumentos para pensar que hay quien aún no sabe qué es una competencia. Disponemos de ejemplos concretos en los que se describen competencias que no son tales. La tabla IV ilustra varias falsas competencias transcritas del Boletín Oficial del Estado. Siendo que esto es así, se hace preciso que todo el ámbito universitario, tanto profesores como gestores, tengan claro qué es una 'competencia' y que utilicen el término correctamente. Una definición de competencia debe ser completa e incluir todos los aspectos, de modo que mi definición es:

Competencia es el constructo de orden superior fruto de la adquisición de conocimientos, habilidades, actitudes $y$ valores necesarios para llevar a cabo una función en un contexto determinado.
Si nos atenemos a esta definición, una competencia requiere conocimientos, habilidades, actitudes y valores, pero no es la suma algebraica de todos ellos, sino que la combinación de estos elementos permite adquirir una capacidad de orden superior, más compleja, que habilita desarrollar una acción o acciones que normalmente describiremos con un verbo (conocer, hacer, razonar, etc.) y que suceden en un contexto y geografía concretos, un ambulatorio urbano, un hospital militar de campaña, el domicilio de un paciente, etc.

\section{Competencias y Bolonia}

¿Y qué tendrá que ver Bolonia y su entorno para haber destapado la 'caja de los truenos' de las competencias? Es posible que prudentemente Bolonia no exija desarrollar el proceso educativo basado en competencias, pero no es discutible que Bolonia pide migrar de un modelo expositivo a un modelo funcional. No es discutible que Bolonia pide migrar de una enseñanza centrada en el profesor a una enseñanza centrada en el alumno. No es discutible que Bolonia pide migrar de una enseñanza basada en el programa a una enseñanza basada en resultados. Bolonia, no lo duden, no da alternativa; Bolonia tiene su punto de mira en la aplicación de la formación universitaria de grado y, por tanto, en la formación basada en competencias.

Por ello es necesario e ineludible orientar los currículos en base a competencias y, por tanto, hay que empezar desarrollando el mapa de resultados de aprendizaje.

Los resultados de aprendizaje estarán definidos en función del fin que se persiga. No serán los mismos si se dirigen a formar especialistas que si se dirigen al grado. Tomemos el grado como referencia para continuar esta exposición, ya que la mecánica no difiere mucho.

\section{Resultados de aprendizaje del grado}

Las competencias finales de un grado deben ser claras y concisas. No deben ser más de las necesarias y en todo caso debemos manejar un número posibilista de ellas, teniendo en cuenta qué 
Tabla V. Relación entre competencia y resultados de aprendizaje.

\begin{tabular}{ll}
\hline Competencia & Resultado de aprendizaje $^{\text {a }}$ \\
\hline Diagnosticar y tratar un paciente hipertenso & \multicolumn{1}{l}{ Conoce las bases celulares } \\
\cline { 2 - 2 } & Sabe tomar la tensión arterial \\
\cline { 2 - 2 } & Maneja correctamente la farmacología \\
\hline
\end{tabular}

aEstos cuatro resultados de aprendizaje no pretenden ser exhaustivos, sino servir de ejemplo.

\section{Tabla VI. Competencias iniciales, intermedias y finales}

Competencia final

Diagnosticar y tratar un paciente hipertenso

Competencia intermedia

Realizar una historia clínica

en un paciente hipertenso

Competencia introductoria ${ }^{a}$

Describir los factores determinantes

de la presión arterial

Describir las características e indicaciones de los fármacos hipotensores

a Estas dos competencias introductorias no pretenden ser exhaustivas, sino servir de ejemplo.

medios serán necesarios para que el estudiante las aprenda y cuánto tiempo se requiere para este aprendizaje. Todas las competencias que se definan o todos los resultados de aprendizaje que deriven de dichas competencias han de ser demostrables y evaluables. Una competencia es una acción y, por tanto, requiere un verbo. En algunos casos pueden tener dos verbos. La tabla $\mathrm{V}$ muestra la relación entre competencia y resultados de aprendizaje. De una competencia se pueden desgranar diferentes resultados de apren- dizaje que han de ser evaluables y que pueden pertenecer a diferentes asignaturas o materias $y$, por supuesto, a diferentes cursos.

Un resultado de aprendizaje o una competencia final no se adquieren forzosamente en una asignatura o materia. Lo más probable es que se requieran diferentes asignaturas y materias para facilitar la comprensión de lo que cada asignatura o materia debe contribuir a cada competencia. Además, como es necesario evaluar procesos intermedios, especialmente mediante evaluaciones formativas y también durante la evaluación continuada, conviene descomponer una competencia no sólo en los resultados de aprendizaje, sino también en diferentes componentes competenciales intermedios a lo largo del tiempo (Tabla VI) y que denominamos 'competencias finales', 'competencias intermedias' y 'competencias introductorias'. Con ello no se pretende complicar el proceso, es más, en muchas ocasiones no es necesario disponer de las competencias intermedias y las introductorias. Hay que construirlas en el caso de que una competencia final sea lo suficientemente compleja como para que su descomposición facilite su entendimiento $y$, en definitiva, su proceso de enseñanza/aprendizaje.

En cambio, síresultaes absolutamente necesario desplegar una tabla de especificaciones (Tabla VII) en la que figuren la competencia final, los resultados de aprendizaje y las metodologías o estrategias educativas que se usarán no para cada competencia, sino para cada resultado de aprendizaje. De la misma manera, la tabla de especificaciones debe mostrar, también para cada 
Tabla VII. Relación entre competencia y resultados de aprendizaje (RA).

\begin{tabular}{|c|c|c|c|}
\hline Competencia & RA & Metodología para cada RA ${ }^{a}$ & Evaluación para cada $\mathrm{RA}^{\mathrm{a}}$ \\
\hline \multirow[t]{9}{*}{$\begin{array}{l}\text { Diagnosticar y tratar } \\
\text { un paciente hipertenso }\end{array}$} & Conoce las bases celulares & Clases & Portafolio \\
\hline & Sabe tomar la tensión arterial & Seminarios & Observatorio \\
\hline & $\begin{array}{l}\text { Maneja correctamente } \\
\text { la farmacología }\end{array}$ & Prácticas & Tests \\
\hline & $\begin{array}{l}\text { Comunica los riesgos y } \\
\text { las conductas alimentarias }\end{array}$ & Simulaciones & Ensayos \\
\hline & & Trabajos de campo & Exposiciones \\
\hline & & Trabajos de grupo & Registros clínicos \\
\hline & & Role playing & Trabajos \\
\hline & & Exposición & Publicaciones \\
\hline & & Evaluación formativa & \\
\hline
\end{tabular}

a La metodología y la evaluación son para cada uno de los RA.

Tabla VIII. Componentes del mapa de competencias (tabla de especificaciones).

\begin{tabular}{l}
$\begin{array}{c}\text { Competencias } \\
\text { Resultados de aprendizaje } \\
\text { Metodologías }\end{array}$ \\
Evaluación formativa \\
Evaluación sumativa \\
Evaluación continuada \\
\hline Departamento/s \\
Asignatura/s Profesores
\end{tabular}

resultado de aprendizaje, las metodologías de evaluación adecuadas.

\section{Mapa de competencias}

La descripción adecuada de las competencias y sus correspondientes resultados de aprendizaje, desplegados en una tabla de especificaciones (Tabla VIII) en la que se describan las metodologías o estrategias educativas a utilizar, los tipos de evaluaciones a realizar y la estructura logística necesaria -esto es, los departamentos, las asignaturas y el profesorado necesarios para cada competencia-, configuran el mapa de competencias. 


\section{Bibliografía}

1. Bloom B, et al. Taxonomía de los objetivos de la educación: la clasificación de las metas educacionales: manuales I y II. Buenos Aires: Centro Regional de Ayuda Técnica, Agencia para el Desarrollo Internacional; 1971.

2. Bologna Declaration. The European Higher Education Area. Joint Declaration of the European Ministers of Education (Bologna); 1999. URL http://www.ond.vlaanderen. be/hogeronderwijs/bologna/about/.

3. Conferencia Nacional de Decanos de Facultades de Medicina Españolas. Libro Blanco del Título de Grado en Medicina. URL: http://www.aneca.es/modal_eval/conver_docs-titulos.html.

4. Facultades de Medicina de la UB, UAB, UDL y URV y AQU Catalunya. Competencias profesionales de los Licenciados en Medicina, formados en las Universidades Catalanas; 2004. URL: http://www.aqu-catalunya.org/scripts/ web/listat.asp?cid=Esp\&cat=pubbl\&id $=2409 \&$ res $=10$.

5. General Medical Council. Good medical practice: regulating doctors; 2006. URL: http://www.gmc-uk.org/static/ documents/content/GMC_GMP_0911.pdf. [07.07.2010].

6. General Medical Council. Tomorrow's doctors: outcomes and standards for undergraduated medical education; 2009. URL: http://www.gmc-uk.org/static/documents/ content/TomorrowsDoctors_2009.pdf. [07.07.2010].

7. GMER-IIME. URL: http://www.iime.org/gmer.htm. Institute for International Medical Education. Versión española: Educ Med 2003; 6 (Supl 2).

8. Guilbert JJ. Educational handbook for health personnel. 6 ed. Geneva: World Health Organization; 1987

9. URL: http://www.deptmedicine.utoronto.ca/CanMEDS/. [27.08.2010].

10. Learning outcomes for the medical undergraduate in Scotland: a foundation for competent and reflective prac- titioners. 3 ed. URL: http://www.scottishdoctor.org/resources/scottishdoctor3.doc. [31.07.2009].

11. MEDINE. The Tuning Learning Outcomes/Competences for Primary Medical Degrees in Europe; 2007. URL: http:// www.tuning-medicine.com. [31.07.2010].

12. Orden ECI/332/2008, de 13 de febrero, por la que se establecen los requisitos para la verificación de los estudios universitarios oficiales que habiliten para el ejercicio de la profesión de médico. BOE n. ${ }^{\circ}$ 40, 15 de febrero de 2008. p. 8351-5.

13. Pales J, et al. Defining the learning outcomes of graduates from the medical school at the University of Barcelona (Catalonia, Spain). Med Teach 2004; 26: 239-43.

14. Palés J. Planificar un currículum o un programa formativo. Educ Med 2006; 9: 59-65.

15 Prat J, Carreras J, Branda L, Miralles R, Fenoll RM, Rodríguez $\mathrm{S}$, et al. Competències professionals bàsiques comunes dels llicenciats em Medicina formats a les universitats de Catalunya. Barcelona: Agència per a la Qualitat del Sistema Universitari de Catalunya; 2004.

16. Smith SS, Dollase R. AMEE guide no. 14. Outcome-based education: Part 2. Planning, implementing and evaluating a competency-based curriculum. Med Teach 1999; 21: 15-22.

17. Spady W. Organizing for results: the basis of authentic restructuring and reform. Educ Leadersh 1988; 46: 4-8.

18 The Association of Faculties of Medicine of Canada. The future of medical education in Canada: a collective vision for MD education; 2010. URL: http://www.afmc.ca/fmec. [29.06.2010].

19. Universitat de Barcelona, Facultat de Medicina. Competències que han d'adquirir els estudiants de medicina durant els estudis de pregrau a la Facultat de Medicina de la Universitat de Barcelona. Barcelona: UB; 2003.

20. Wojtczak A, Schwarz MR. Minimum essential requirements and standards in medical education. Med Teach 2000; 22: 555-63. 